\title{
Non-recursive complexity reduction encoding scheme for performance enhancement of polar codes
}

\author{
${ }^{1}$ Prakash K M, ${ }^{2}$ Dr. G S Sunitha \\ ${ }^{1}$ Assistant Professor, Dept. of E\&C, \\ Bapuji Institute of Engineering and Technology, Davangere, Karnataka, India, \\ ${ }^{2}$ Professor and Head,Dept. of E\&C, \\ Bapuji Institute of Engineering and Technology, Davangere, Karnataka, India \\ ²prakashk_m@rediffmail.com,2drsunitha_r_c@yahoo.com
}

Abstract-Recent advancement in digital communication application has grown significantly. Developed communication methods demand for higher performance for real time application scenarios where noisy channels are present. In this context, polar codes have grown as a promising technique for errorcorrecting scheme and performance enhancement in memoryless channel communication scenarios. This article presents a brief study about polar coding, principle, encoding schemes and introduces a new encoding scheme for performance improvement of polar codes.

In this work, we propose new encoding schemes which mainly address the issue of computational complexity and memory requirements for polar coding scheme. Here we address the issue related to systematic polar coding schemes and their computational complexity reduction. Proposed method computes the requirement of XOR implementation to encode the data and total memory requirement for complete encoding scheme. An experimental study is carried out to show the significance of proposed model where performance is computed in terms of bit error rate and frame error rate. Experimental study shows that proposed systematic encoding scheme outperform when compared to state-of-art technique.

\section{INTRODUCTION}

During recent years, demand of digital communication has grown rapidly which has improved the emerging technique coding theory for efficient data communication. In this field of data coding, Shannon's introduced channel coding theory which is best known effort for transmission of data reliability over a wireless noise channel and performance of system can be enhanced. Mainly, channel coding theory depends on the essential ideas which are:

Code selection: Transmittedcode word and received data equipartition property while performing for large number of code length

Maximum likelihood decoding: Asymptotic equipartition probability (AEP) is a promising technique which guarantees for better communication by reducing error in received codeword. However, random code selection is also an efficient technique by considering mathematicalcomplexity during data encoding and decoding. In coding theory, achieving better coding performance is still remains a challenging issue for researchers. In order to address this issue various techniques have been proposed such as LDPC (Low-Density Parity Check) [1], turbo coding[2] etc. Another challenging issue is known as practical implementation of these coding schemes for real time application scenarios.

In coding theory, coding randomness is an issue which affects the performance of communication which is introduced in turbo code due to interleavers connected between check nodes and variable nodes while considering LDPC. In order to achieve reliable and efficient decoding, turbo codes utilized BCJR algorithm and LDPC uses belief propagation approach. These methods improve the performance and due to better performance requirements it is adopted in WCDMA, LTE and 3GPP standards. These methods still suffer from the capacity to achieve joint AEP when communication is performed over noisy channels.

In today’s scenario, this issue addressed by a new approach called as polar codes, proposed by Arikan [3]. This is a new approach for designing error-correcting coding scheme to achieve better capacity in a binary input discrete memoryless channel (B-DMC). This approach enhanced the coding scheme by utilizing polarization scheme according this scheme; independent channels are portioned into two categories: Good channel and Bad Channel. After achieving the complete code length, channels are synthesized into two categories as: noisy and noise free. In order to reduce the complexity, successive cancellation decoding approach is proposed by Lin et al [1] where and efficient decoder architecture is developed for non-binary LDPC codes with the help of Min-Sum algorithm. SC decoders perform estimation of bit message by applying recursive architecture on polar code. This decoding is a step wise decision process which does not depend on the previous steps. 
Recently, lots of works have been done in polar coding to achieve the efficient performance in memoryless communication channels with the help of successive cancellation decoding. During data transmission over a symmetric channel,polar codes don't induces any error which is main parameter to improve the performance of polar code[4]. Sarkis et al [5] presented a new scheme for fast polar coding by considering different rate of coding and fixed code length. Work presented in [5] addressed the issue of throughput and speed of polar codes by using successive cancellation decoding. Another similar approach is discussed in [6] which is based on polar code performance improvement strategy, implemented using CMOS technology.

Initially, polar codes introduced as non-systematic blocks according to the study present in [3]. Due to improved performance requirements, systematic polar codes were developed as described in [7]. For fast encoding scheme, polar codes have been proven significant techniques as discussed in [5]. In this work, we present a new approach for systematic polar encoding to improve the efficiency of data encoding by reducing the number of XOR gate requirements. Proposed approach addresses the issues of memory requirement and solves it by estimating the total number of XOR requirement for computation.

Rest of the article is arranges as follows: section II describes recent works presented in the field of polar encoding scheme, proposed model is described in section III, section IV present detailed analysis of experimental study and results, finally, concluding remarks are presented in section V.

\section{RELATED WORK}

This section provides brief description about most recent studies presented in this field of coding theory for digital communication by considering polar codes. In order to reduce the complexity in polar coding schemes which are based on belief propagation technique, early stopping is discussed in [8] where early stopping is performed by discarding unnecessary iterations during computation.

Zhao et al [9] discussed the significance of polar codes and presented a new method for digital communication coding theory for memory less broadcast channels. This method is called as Blackwell channels which considers noise during the communication period. This scheme uses marton coding theory which uses auxiliary random variables for providing inputs to the channels.Ullah et al.[10] presented data coding scheme for MIMO networks by utilizing LDPC based approach. This approach mainly aims on correction of magnitude of message by using LDPC min-sum decoding algorithm. According to this method, if any sign change occurs in the data then the original message is modified by using a normalization factor. This work aims on the performance improvement of MIMO system by incorporating QC LDPC method for flat fading channel scenario. Performance of digital communication by considering AWGN channel is addressed in [11].

In [12], authors addressed the issue of implementation complexity of polar codes. In order to solve this issue, hardware based implementation is presented by considering systematic polar encoding and decoders. Proposed architecture can perform polar encoding for any code length and varied rate. This method shows significant improvement in complexity reduction, fast computation and flexibility during encoding.

In [13], non-binary polar codes are discussed and performance analysis is carried out for memorylesschannels using rate assignment theory. According to this method, memory less channels can be polarized into multiple levels for performance improvement. In order to perform rate assignment strategy an element-wise exchange (EWEX) method is developed which aims on minimizing the BLER performance of system. Key challenge in this work is experienced in terms of complexity during data transmission, where input data is increasing then the complexity of this method increases. As we have discussed that achieving better throughput is a crucial task in this field. In order to cater this issue, Lin et al. [14] presented a new decoding scheme for polar codes using CMOS technology. This work includes cyclic redundancy check and SCL decoding scheme. In contrast, it is presented that existing CRC schemes suffers from the issue of latency and throughput performance. This issue is solved in this work by using reduced latency list decoding (RLLD) scheme using a polar code based approach. According to this work, a tree is formed over decoding which helps to achieve the coded bits using polar code

\section{SYSTEM MODEL}

This section provides system modeling for polar code construction for systematic polar encoding.As discussed before that polar codes are binary codes in nature which are defined by a triple such as $(\mathcal{N}, \mathcal{K}, \mathcal{J})$, where $\mathcal{N}$ denotes code length, message length is denoted by $\mathcal{K}$ and $\mathcal{J}$ denotes information bit indices which can be expressed as $\mathcal{J} \subseteq \mathcal{N},|\mathcal{J}|=\mathcal{K}$. Remaining indices $(\mathcal{N}-\mathcal{K})$ are known as frozen bits.

Figure 1 shows overall architecture of polar encoding scheme. According to the working of polar code, initially parameters are defined for polar code construction such as code rate; code length etc. in the next stage, polar code construction is applied by applying Bhattacharya formulation. After finishing construction of polar code, data encoding is applied which results in data mapping into another format for transmission. During data transmission, AWGN channel is constructed and encoded data is modulated with the help of binary phase shift keying modulation scheme. At the receiver end, data is received and performance is computed in terms of frame error rate and bit error rate. 


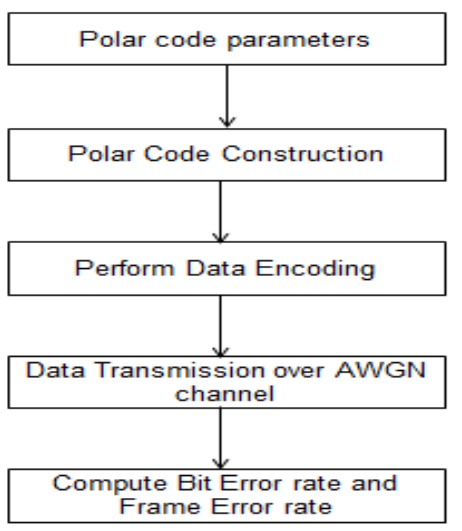

Fig.1. System Architecture of polar encoding and decoding

Let $(\mathcal{N}, \mathcal{K}, \mathcal{J})$ be a polar code and generator matrix is denoted as $\mathcal{G}=\left(\mathbb{F}^{\otimes n}\right)_{\mathcal{J}}$ where $n \triangleq \log _{2}(\mathcal{N})$, with the help of this codeword $x$ is generated with a message vector $u$ of $\mathcal{K}$ information bits which is expressed as follows:

$$
x=u \cdot \mathcal{G}=d \cdot \mathbb{F}^{\otimes n}
$$

Where $d$ represents a vector of $\mathcal{N}$ bits which has relationship with codewords and contains other constraints which are denoted as $d_{\mathfrak{\jmath}}=u, d_{\mathfrak{\jmath} c}=0$ and $\mathcal{J}^{c} \triangleq \mathcal{N} / \mathfrak{\jmath}$. Here $d_{\mathfrak{\jmath} c}$ are known as frozen bits which are set to zero.

In this work, main aim is to develop a systematic polar code which can be expressed similarly as presented in (1), however, according to systematic polar codes, message vectors are mapped into codewords. Let us consider indices of $\mathcal{K}$ bits present in codeword $x$ where message bits are ambiguously visible. According to the study presented in [7]these set can be selected equal to the set of information bit indices $\mathcal{J}$. In general, in a systematic polar code it is considered that message bits are appeared as first $\mathcal{K}$ bits which motivate to develop an efficient systematic polar encoding scheme which can provide solution to information $u$ of $\mathcal{K}$ bits, expressed as:

$$
x=y \cdot \mathbb{F}^{\otimes n}
$$

In expression (2), it is considered that $y_{\jmath}$ and $x_{\mathcal{J}}$ are unknowns. In a systematic polar codeword $x$, information bits and parity bits are denoted as $x_{\mathfrak{J}}=u$ and $x_{\mathfrak{J}}$ respectively. For an illustrative example if value of $(\mathcal{N}, \mathcal{K}, \mathcal{J})=(4,2,\{1,3\})$ and $u=[1,0]$, replaced in (2) then it can be expressed as

$$
\left[1, x_{2}, 0, x_{4}\right]=\left[y_{1}, 0, y_{3}, 0\right]\left[\begin{array}{llll}
1 & 0 & 0 & 0 \\
1 & 1 & 0 & 0 \\
1 & 0 & 1 & 0 \\
1 & 1 & 1 & 1
\end{array}\right]
$$

As discussed before that $x_{2}, x_{4}, y_{1}, y_{3}$ are unknowns, number of unknowns are equal to $\mathcal{N}$ which are shared between $x$ and $y$.According to Arrkan, this approach has more complexity which cannot be achieved by $\Theta(\mathcal{N} \log \mathcal{N})$ XOR implementation. In order to reduce the complexity issue, here we propose a new systematic polar code scheme.

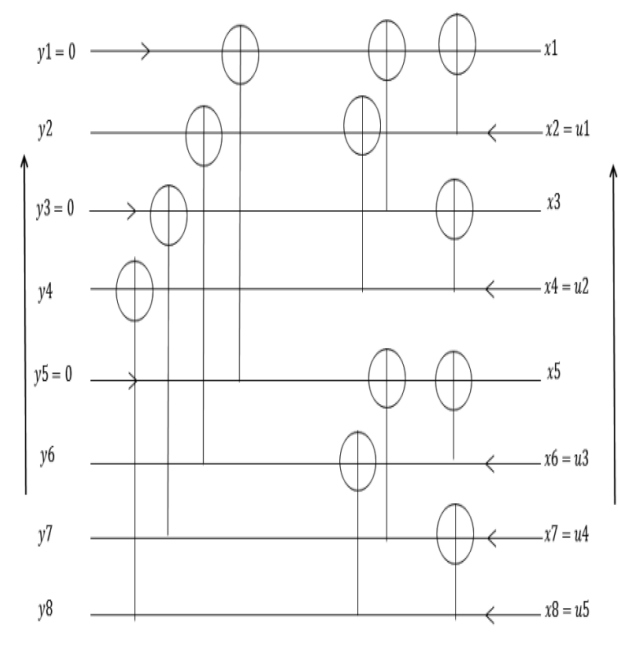

Fig.2. Systematic polar encoding graph 
Proposed scheme for Systematic Polar code

This section describes about proposed approach for systematic polar encoding scheme which can provide solution for (2) efficiently. Proposed approach aims on three main constraints during implementation such as:

- Least number of XOR

- Splitting encoder equations

- Computation of exact number of XORsand memory for implementation

In order to perform encoding, a new encoder is developed which is non-recursive in nature and meets the constraints which are required for efficient performance. According to systematic polar code based on (2), unknown and known bits distribution is depicted in figure 2 which is the proposed circuit for polar encoding. In this proposed architecture, $\mathcal{N}\left(1+\log _{2} \mathcal{N}\right)$ indices are used to store the each bit information . Horizontal connections presents $(n+1)$ nodes. Further, out of $(n+1)$ nodes only 1 node is known at one end of the circuit. Calculation is initialized from known end and gradually incremented toward the other end of circuit. It can be observed from figure 2 that any of $(\mathrm{n}+1)$ node computation at horizontal connection includes nodes for computation which belongs from same connection and nodes which are below to this node and horizontal connection must be evaluated as bottom-up approach. This approach utilizes $\frac{\mathcal{N}}{2} \log _{2} \mathcal{N}$ XORs and contains $\mathcal{N}\left(1+\log _{2} \mathcal{N}\right)$ bits. Working process of proposed encoding is presented in algorithm 1 which is as follows:

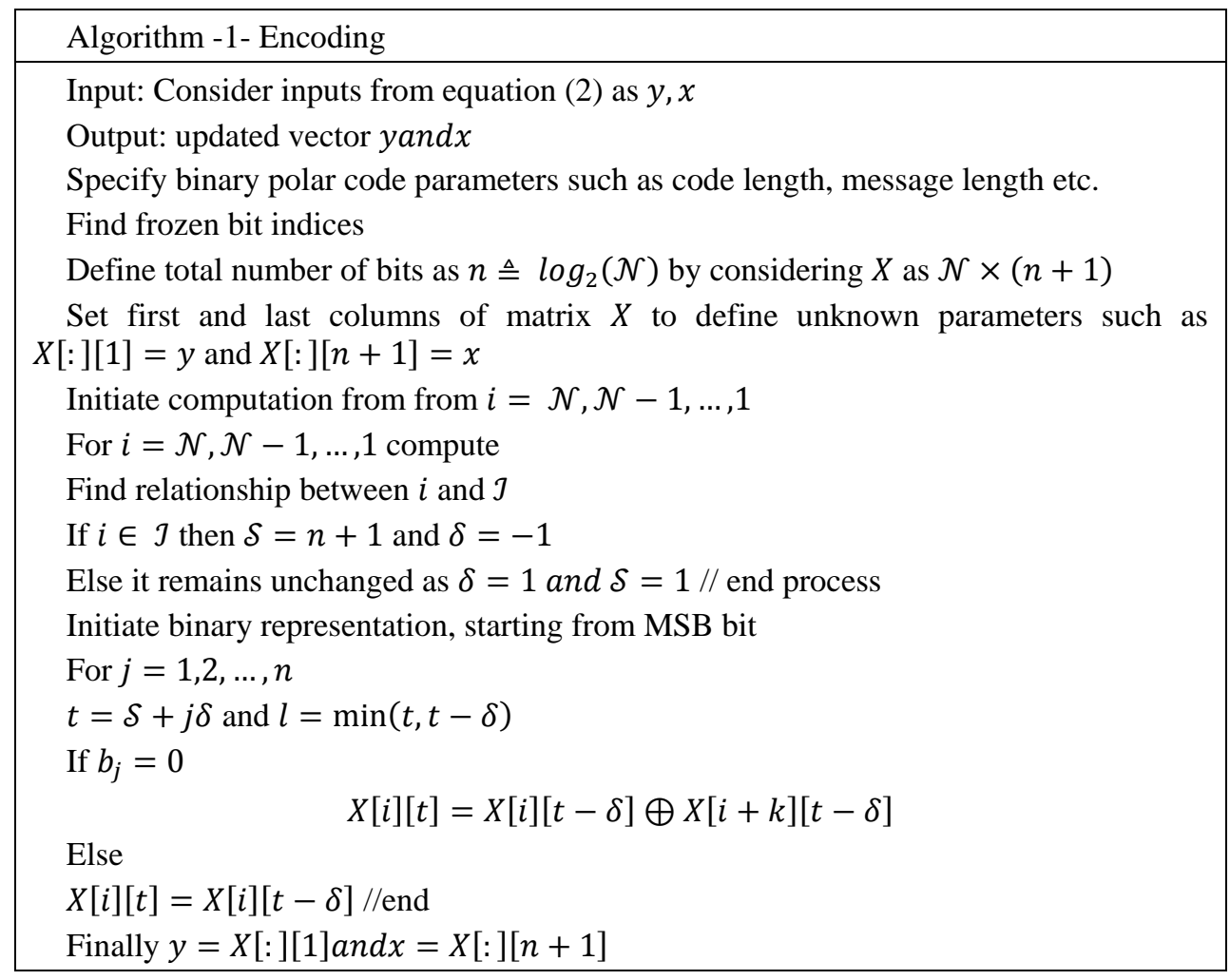

\section{RESULTS AND DISCUSSION}

In this section detailed performance of proposed polar encoding scheme is presented by varying the simulation parameters. Initial case study is conducted by considering polar code which has code length as 256 and code rate is $\frac{1}{2}$ over an additive white Gaussian noisy channel. During transmission, BPSK (Binary Phase Shift Keying)modulation scheme is used

In order to map the codeword $x \in \mathbb{F}_{2}^{N}$ in a BPSK signal, following rule is used

$$
s_{i}=\left\{\begin{array}{l}
+1 \text { for }_{i}=0 \\
-1 \text { for }_{i} \neq 0
\end{array}\right.
$$

Where $s \in\{-1,+1\}^{N}$, by considering this assumption, AWGN channel is expressed as follows:

$$
y=s+z
$$

Where $z$ is an identical distribution of Gaussian variables which is having zero means and variance value as $\frac{N_{0}}{2}$. Simulation parameters are given in table 1. 
Table.1. Simulation parameters

\begin{tabular}{|c|c|}
\hline Simulation Parameter & Considered Value \\
\hline Code length & 128,256 \\
\hline Code rate & $1 / 2$ \\
\hline Range of SNR & -1 to 5 \\
\hline Design SNR Requirement & 0 \\
\hline Monte Carlo size & 10000 \\
\hline
\end{tabular}

In order to evaluate the performance, we have performed two case studies by considering code length as 128 and 256. Performance of proposed model is computed in terms of frame error rate and bit error rate which is compared with existing systematic polar code performance. Figure 3 shows the performance of scenario 1 in terms of bit error rate, where code length is 128 and code rate 0.5 and compared with the performance of systematic and non-systematic polar code methods.

In scenario 1 we have considered the bit error and frame error rate for code length 128 and it is represented in Fig 3 and Fig 4.

In scenario 2 we have considered the bit error and frame error rate for code length 256 and it is represented in Fig 5 and Fig 6.

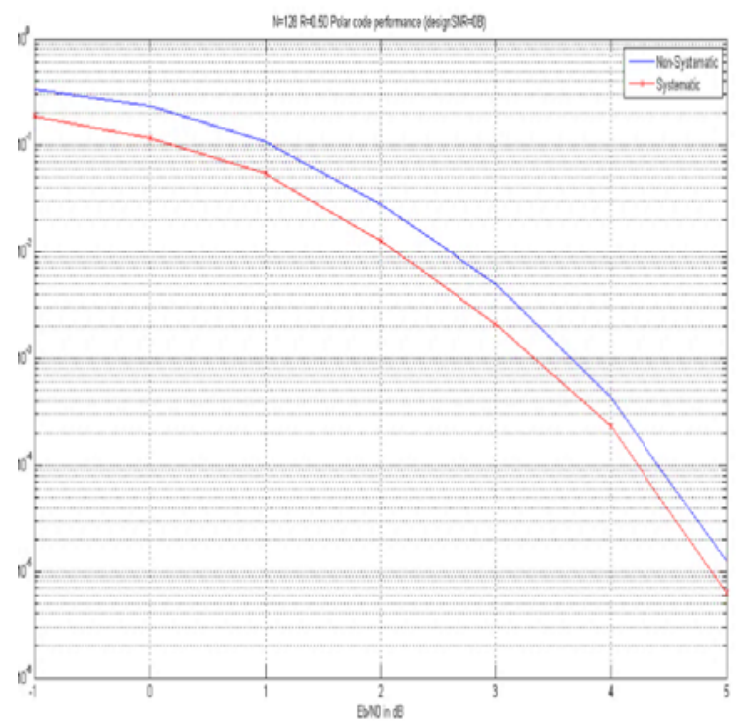

Fig.3. Bit error rate performance for scenario 1

Similarly, performance of proposed polar encoding performance in terms of frame error rate is presented in figure 4 for scenario 1.

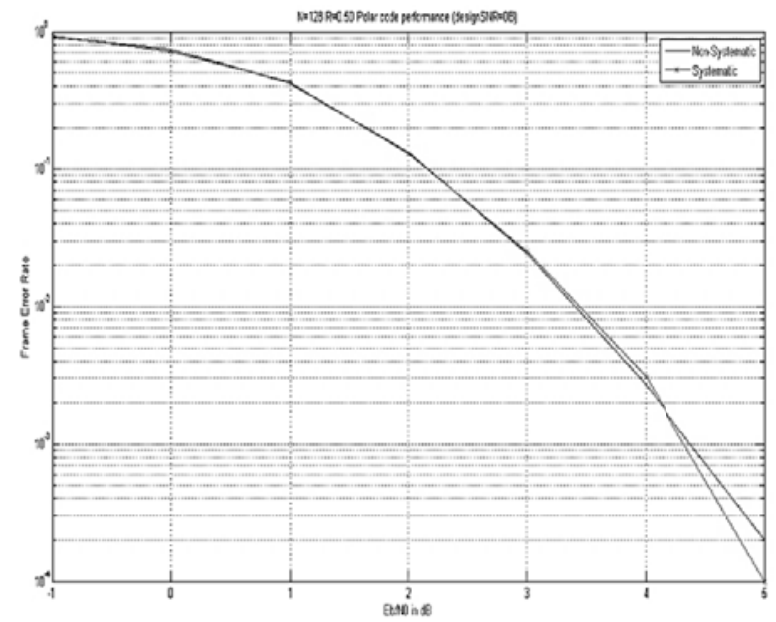

Fig.4. Frame Error rate performance for scenario 1 
As discussed before, here we consider second scenario for performance evaluation where code rate is $1 / 2$ and code length as 256. First of all, performance is computed in terms of bit error rate as presented in figure 5.

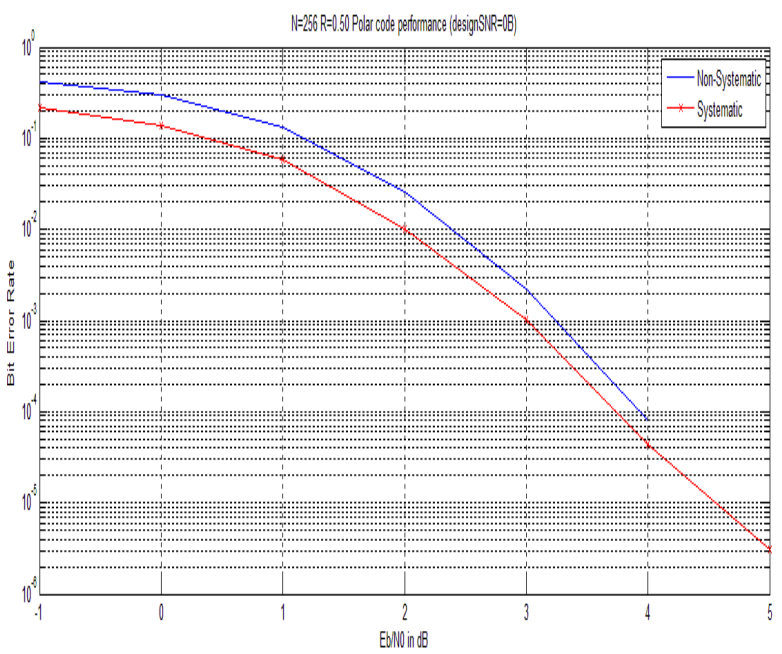

Figure.5. Bit error performance for scenario 2

In figure 6, frame error rate performance is presented by considering varied SNR rate at design SNR requirement as $0 \mathrm{~dB}$.

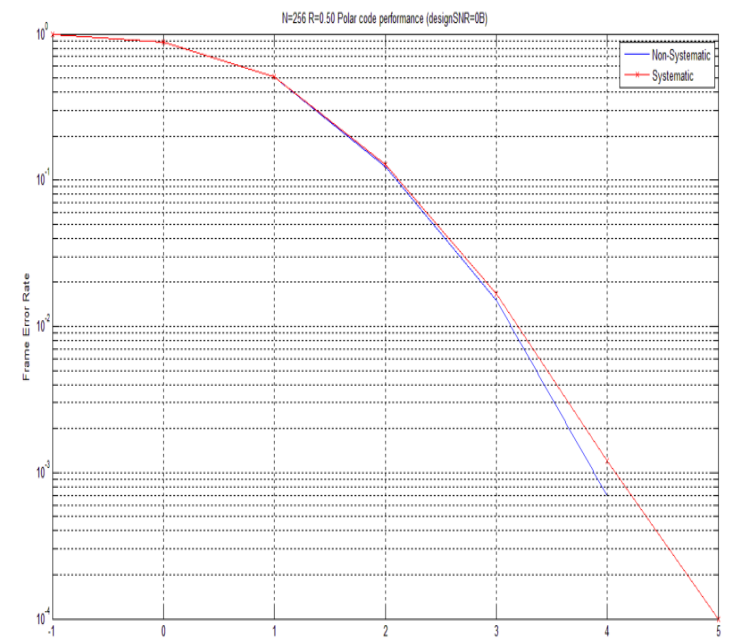

Fig.6. Frame Error rate performance considering various SNR for scenario 2

From the study presented,in this section it can be concluded that the proposed model performs better when compared to non-systematic polar encoding scheme. A comparative analysis of bit error rate, frame error rate is presented in table 2 and 3 for simulation scenario 1 and 2.

Table. 2. Non Systematic Polar code performance evaluation

\begin{tabular}{|c|c|}
\hline Frame Error Rate & Bit Error Rate \\
\hline 0.915 & 0.3397 \\
\hline 0.736 & 0.2373 \\
\hline 0.421 & 0.1101 \\
\hline 0.1321 & 0.0277 \\
\hline 0.0245 & 0.005 \\
\hline 0.0027 & 0.0004 \\
\hline 0.0002 & 0 \\
\hline
\end{tabular}


Table 2 shows performance analysis of proposed model for non-systematic polar encoding by considering frame error rate and bit error rate for varied SNR requirements. For this analysis SNR is varied from -1 to 5 and performance is computed where it is achieved that frame error rate and bit error rate decreases when SNR is increasing. Another similar scenario is presented in table 3 as proposed model which shows performance of systematic polar encoding by using proposed encoding scheme.

Table. 3. Systematic Polar code performance evaluation

\begin{tabular}{|c|c|}
\hline Frame Error & BER \\
\hline 0.929 & 0.1891 \\
\hline 0.722 & 0.1171 \\
\hline 0.426 & 0.0547 \\
\hline 0.13 & 0.0125 \\
\hline 0.0253 & 0.0021 \\
\hline 0.0031 & 0.0002 \\
\hline 0.0001 & 0 \\
\hline
\end{tabular}

\section{CONCLUSION}

In this work, our main aim is to study about polar codes and develop an efficient encoding scheme to reduce the computational complexity in polar encoding. In order to do this, a non-recursive encoding scheme is developed for systematic polar codes by considering the complexity of $\Theta(\mathcal{N} \log \mathcal{N})$. Proposed encoding study performs encoding for any given arbitrary frozen bit indices. Proposed encoding scheme aims on complexity reduction of polar code by estimating the number of XOR implementation and memory requirement for polar encoding. Experimental study of proposed model shows significance improvement in the performance in terms of bit error rate and frame error rate when compared to existing models.

\section{REFERENCES}

[1] C. L. Lin, S. W. Tu, C. L. Chen, H. C. Chang and C. Y. Lee, "An Efficient Decoder Architecture for Nonbinary LDPC Codes With Extended Min-Sum Algorithm," in IEEE Transactions on Circuits and Systems II: Express Briefs, vol. 63, no. 9, pp. 863-867, Sept. 2016.

[2] L. H. Abderrahmane, "Design of a new interleaver using cross entropy method for turbo coding," in IET Communications, vol. 7, no. 9, pp. 828-835, June 112013.

[3] E. Arlkan, "Channel polarization: A method for constructing capacity achieving codes for symmetric binary-input memoryless channels,” IEEE Trans. Inf. Theory, vol. 55, no. 7, pp. 3051-3073, 2009.

[4] R. U. Marco Mondelli, S. Hamed Hassani, "Unified scaling of polar codes: Error exponent, scaling exponent, moderate deviations, and error floors,” CoRR, vol. abs/1501.02444, 2015. [Online]. Available: http://arxiv.org/abs/1501.02444

[5] G. Sarkis, P. Giard, A. Vardy, C. Thibeault, and W. J. Gross, "Fast polar decoders: Algorithm and implementation,” IEEE J. Sel. Areas Commun., vol. 32, no. 5, pp. 946-957, May 2014

[6] Y. S. Park, Y. Tao, S. Sun, and Z. Zhang, “A 4.68Gb/s belief propagation polar decoder with bit-splitting register file,” in Symp. on VLSI Circuits Dig. of Tech. Papers, Jun 2014, pp. 1-2.

[7] E. Arıkan, “Systematic polar coding,” IEEE Commun. Lett., vol. 15, no. 8, pp. 860-862, 2011.

[8] C. Simsek and K. Turk, "Simplified Early Stopping Criterion for Belief-Propagation Polar Code Decoders," in IEEE Communications Letters, vol. 20, no. 8, pp. 1515-1518, Aug. 2016.

[9] L. Zhao, S. I. Choi and S. Y. Chung, "Polar Code Design for Noisy Blackwell Channels," in IEEE Communications Letters, vol. 20, no. 8, pp. 1495-1498, Aug. 2016.

[10] Waheed Ullah, Yang Fengfan, Abid Yahya,” QC LDPC Codes for MIMO and Cooperative Networks using Two Way Normalized Min-Sum Decoding”, TELKOMNIKA Indonesian Journal of Electrical Engineering Vol 12, No 7

[11] Xue Jianbin, Li Songbai. Transmission Performance Research of Digital Modulation Signals in AWGN Channel. TELKOMNIKA Indonesian Journal of Electrical Engineering. 2013; 11(2): 991-997.

[12] G. Sarkis, I. Tal, P. Giard, A. Vardy, C. Thibeault and W. J. Gross, "Flexible and Low-Complexity Encoding and Decoding of Systematic Polar Codes," in IEEE Transactions on Communications, vol. 64, no. 7, pp. 2732-2745, July 2016.

[13] D. Wu, Y. Li and Y. Sun, "Rate assignment for multi-level polarised non-binary polar codes," in IET Communications, vol. 10, no. 10, pp. 1151-1155, 712016.

[14] J. Lin, C. Xiong and Z. Yan, "A High Throughput List Decoder Architecture for Polar Codes," in IEEE Transactions on Very Large Scale Integration (VLSI) Systems, vol. 24, no. 6, pp. 2378-2391, June 2016. 